\title{
Funcionalidades para modelo de aplicativo de smartphone para prevenção do comportamento suicida
}

\author{
Functionalities for smartphone application model for suicide behavior prevention \\ Funcionalidades para modelo de aplicación de smartphone para la prevención del comportamiento \\ suicida
}

Recebido: 21/05/2021 | Revisado: 29/05/2021 | Aceito: 02/06/2021 | Publicado: 18/06/2021

Sarah Soares Barbosa

ORCID: https://orcid.org/0000-0002-6847-3942

Escola de Saúde Pública de Florianópolis, Brasil

E-mail: esf.sarah@gmail.com

Jeferson Rodrigues

ORCID: https://orcid.org/0000-0002-8612-9088 Universidade Federal de Santa Catarina, Brasil Departamento de Enfermagem

E-mail: jeferson.rodrigues@ufsc.br

Gustavo Ferreira Guimarães

ORCID: https://orcid.org/0000-0003-0111-9663 Universidade Federal de Santa Catarina, Brasil

E-mail: gutiguim@gmail.com

Jean Everson Martina

ORCID: https://orcid.org/0000-0003-4104-1741 Universidade Federal de Santa Catarina, Brasil

E-mail: jean.martina@ufsc.br

Sara Mendes Boeira Lopes

ORCID: https://orcid.org/0000-0002-0360-0234

Universidade Federal de Santa Catarina, Brasil

E-mail: saramblopes@gmail.com

\begin{abstract}
Resumo
Introdução: o comportamento suicida é um problema de saúde pública, portanto é essencial a oferta de recursos para prevenção e amparo da pessoa em sofrimento Objetivo: identificar funcionalidades em aplicativos de smartphone internacionais para criação de aplicativo brasileiro para prevenção do comportamento suicida. Método: revisão de aplicativos de smartphone, identificação de funcionalidades manualmente, envio das funcionalidades encontradas a especialistas para avaliação da aplicabilidade em protótipo. Resultados e Discussão: das 71 funcionalidades avaliadas, 14 obtiveram consideração de $80 \%$ dos experts como adequadas ao protótipo. Destas, três foram consideradas adequadas por $100 \%$ dos avaliadores. Oito são consideradas funcionalidades de autogestão do cuidado e 6 de gestão compartilhada com serviços e/ou profissionais de saúde. Conclusão: identificadas 14 funcionalidades na revisão integrativa de literatura, e consulta à especialistas na prevenção ao suicídio. Indicou-se modelo para posterior desenvolvimento de aplicativo de smartphone para a prevenção do comportamento suicida no Brasil. Por fim, reconhece-se que este aplicativo poderá fazer parte de pesquisas futuras sobre a prevenção do comportamento suicida.

Palavras-chave: Suicídio; Aplicativos móveis; Saúde mental; Tecnologia.
\end{abstract}

\begin{abstract}
Introduction: suicidal behavior is a public health issue, therefore, it's essential to offer resources for threats and support people suffering. Objective: to identify international smartphone app's functionalities for the creation of a Brazilian smartphone app for the suicidal behavior prevention. Method: donwloading smartphone app's, identifying functionalities manually, sending the functionalities found to specialists for evaluating evaluation of applicability in prototype smartphone app. Results and Discussion: of the 71 functionalities evaluated, 14 obtained $80 \%$ of the specialists' consideration as suitable to the prototype. Of these, three were were considered suitable for $100 \%$ of the evaluators. Eight are self-managed care functionalities and 6 are shared management with services and /or health professionals. Conclusion: 14 functionalities were identified in the integrative literature review and consultation with experts in suicide prevention. Was indicated a model for further development of a smartphone app to prevent suicidal behavior in Brazil. Finally, it is recognized that this app may be part of future research on the prevention of suicidal behavior.
\end{abstract}

Keywords Suicide; Mobile applications; Mental health; Technology. 


\begin{abstract}
Resumen
Introducción: el comportamiento suicida es problema de salud pública, por tanto, es esencial ofrecer recursos para prevencióny y apoyar las personas que sufren. Objetivo: identificar funcionalidades de aplicaciones internacionais de smartphone para creación de aplicación brasileña para prevención del comportamiento suicida. Método: descargar aplicaciones de smartphone, identificar funcionalidades manualmente, enviar las funcionalidades encontradas a especialistas para evaluación de aplicabilidad en prototipo. Resultados y discusión: de las 71 funcionalidades evaluadas, 14 obtuvieron $80 \%$ de consideración de los especialistas como adecuada para el prototipo. De estos, tres se consideraron adecuados per 100\% de los evaluadores. Ocho son funcionalidades de atención autogestionadas y 6 son gestión compartida con servicios y / o profesionales de la salud. Conclusión: se identificaron 14 funcionalidades en revisión integral de literatura y consulta con expertos en prevención del suicidio. Se indicó un modelo para desarrollo futuro de aplicación de smartphone para prevenir el comportamiento suicida en Brasil. Por último, se reconoce que esta aplicación puede ser parte de futuras investigaciones sobre la prevención de la conducta suicida.
\end{abstract}

Palabras clave: Suicidio; Aplicaciones móviles; Salud mental; Tecnología.

\title{
1. Introdução
}

O suicídio pode ser explicado como toda a morte que resulta mediata ou imediatamente de um ato positivo ou negativo realizado pela própria vítima (Durkheim, 1977). Entretanto, esta ação envolve aspectos além do ato suicida em si, uma vez que existem três diferentes categorias que envolvem o comportamento suicida (Werlang, Borges, \& Fensterseifer, 2005).

A ideação suicida, inicialmente, é um dos indicadores para o risco de suicídio e pode ser o primeiro passo para a sua efetivação. Em seguida, podem estar presentes as ideias ou pensamentos de morte, somadas a ameaças, gestos ou tentativas de suicídio e, por fim, há o suicídio em si (Botega, 2015). O suicídio é considerado o ato pelo qual uma pessoa procura tirar sua vida, estando ciente, ou não, das consequências de sua ação, ou, todo ato pelo qual a pessoa causa lesão a si mesmo, independente do grau de intenção letal e do verdadeiro motivo desse ato (Padurariu et al., 2016; Botega, 2015), mais especificamente, trata-se da morte por lesão autoprovocada, através de um ato voluntário/involuntário contra a própria vida, resultando em morte (Werlang, Borges, \& Fensterseifer, 2005).

Uma vez que este caracteriza um problema de saúde pública, é importante que se elenquem modos de prevenção ao comportamento suicida, para que se possa auxiliar a pessoa em sofrimento. Para Botega (2009) e a Organização Mundial da Saúde (2006), para que se possa prevenir o comportamento suicida deve-se, principalmente: focar no apoio e melhoria dos contextos interpessoais e sociais; diminuir significativamente as condições de riscos emocionais, físicas e econômicas; identificar as pessoas que estão em risco e que a ele são vulneráveis; entender as circunstâncias que influenciam o seu comportamento autodestrutivo e estruturar intervenções eficazes (Botega, 2009; World Health Organization [WHO], 2006).

Nesse sentido, sabe-se que alguns fatores são considerados protetores para o risco de suicídio, entre eles: apoio da família e amigos, crenças religiosas, culturais ou étnicas, integração social e envolvimento na comunidade, uso do tempo com lazer e também acesso aos serviços de saúde mental. Entretanto, ainda esses fatores protetivos possam equilibrar circunstâncias difíceis na vida, sabe-se que eles não eliminam totalmente o risco do suicídio (WHO, 2006).

Colaborando com esses fatores protetivos estão os aplicativos móveis para celular, recursos que estão disponíveis aos usuários que possuem smartphones e são acessíveis sempre que a pessoa necessitar de ajuda. A OMS indica que as intervenções via aplicativos móveis de celular podem ser úteis em países em desenvolvimento, por exemplo, onde o apoio às pessoas com risco de suicídio pode ser limitado. Desse modo, a organização faz recomendações acerca do uso de dispositivos móveis, considerando estes uma opção para apoio e tratamento das pessoas em risco de suicídio, uma vez que se sabe que os aplicativos de celulares tem potencial de atingir um grande número de pessoas através do uso de dispositivos eletrônicos e que podem ser implementados a um baixo custo (World Health Organization [WHO], 2014).

Dentro desses apps, podem-se incluir recursos que protejam e apoiem a pessoa em momentos que se encontrar em crise. Entre esses recursos, podem-se incluir, por exemplo, certas formas de diário para o usuário, pesquisas de sintomas 
psiquiátricos, GPS (Global Positioning System) que auxilie o usuário a encontrar um serviço de saúde mais próximo e até

indicar a localização de uma emergência de saúde e/ou psiquiátrica para as pessoas que se sentem em risco iminente (Band, 2013; Fjeldsoe, Marshall, \& Miller, 2009; Boulos, Wheeler, Tavares \& Jones, 2011; Pop-Eleches et al., 2011).

Observando, portanto, a potencialidade desses dispositivos móveis para auxílio na prevenção do comportamento suicida, percebe-se a importância do rigor na construção desses produtos, ou seja, é fundamental que os recursos oferecidos sejam baseados em literatura científica e/ou experiências anteriores de sucesso, buscando oferecer o melhor acesso ao usuário e preservando a segurança e a manutenção à vida do mesmo.

Diante do exposto, questiona-se: quais funcionalidades podem ser propostas para o desenvolvimento de um protótipo de aplicativo de celular para a prevenção do comportamento suicida?

O objetivo deste estudo foi identificar funcionalidades existentes em aplicativos internacionais que podem ser consideradas adequadas para um protótipo de aplicativo de celular para a prevenção do comportamento suicida.

\section{Metodologia}

Trata-se de estudo desenvolvido inicialmente a partir de uma Revisão Integrativa da Literatura, que se trata de um método de pesquisa que possibilita a organização sistemática e a síntese de evidências disponíveis acerca do tema a ser estudado. Para a elaboração da mesma, a análise dos estudos incluídos deve ser realizada de forma dinâmica e incluir estudos teóricos, empíricos e permitir reuni-los para que se construa uma conclusão geral a partir dos resultados de cada estudo (Pompeo, Rossi, \& Galvão, 2009).

Para o desenvolvimento do presente estudo, após a revisão integrativa da literatura acerca do tema, realizou-se uma revisão dos aplicativos de celular encontrados nos estudos selecionados e mapeou-se e descreveu-se cada uma das funcionalidades de cada aplicativo. Esse processo se deu de modo manual, onde se fez o download de cada um dos aplicativos e acessou-se cada item funcional do mesmo, realizando uma descrição das funcionalidades e o que elas oferecem ao usuário. A partir desse mapeamento foi elaborada uma planilha, contendo todas as informações acerca dos apps estudados: o nome do aplicativo, o(s) artigo(s) onde ele foi citado, a app store onde ele se encontrava disponível no momento da busca, o país de desenvolvimento, a data da pesquisa, a versão do aplicativo no momento da busca, o idioma, o número de downloads, a nota média de avaliação, o número de avaliadores, os desenvolvedores, se foi testado em seres humanos, as descrições sobre o app; se é interativo ou informativo; suas funcionalidades. Essas, por sua vez foram organizadas conforme semelhança de objetivos/funções e divididas em 8 categorias.

A etapa seguinte do estudo consistiu em organizar as funcionalidades encontradas em um Formulário Google ${ }^{\mathrm{e}}$ enviá-lo a cinco especialistas na área estudada. Os cinco selecionados analisaram e retornaram o formulário à pesquisadora principal, avaliando quais funcionalidades os mesmos considerassem aplicáveis a um protótipo de app para a prevenção do comportamento suicida a ser desenvolvido no Brasil.

Após o retorno dos formulários pelos especialistas avaliadores, utilizou-se uma ferramenta do Formulário Google ${ }^{\circledR}$ que gera gráficos a partir dos resultados da pesquisa. Foi considerada relevante a funcionalidade que obtivesse a aprovação, ou seja, fosse considerada adequada por no mínimo dois terços dos especialistas, o que resultaria em 3,33 avaliadores. Entretanto, como é necessário utilizar números inteiros nessa situação, foi considerado o número mínimo de quatro experts a considerarem adequadas as funcionalidades mapeadas na revisão, resultando numa porcentagem de $80 \%$ de adequação do item.

Desse modo, foram utilizados os gráficos do programa Formulário Google ${ }^{\circledR}$ para aplicar a seleção de funcionalidades em que no mínimo $80 \%$ dos juízes considerassem adequadas. Esse processo foi feito de forma manual, contando quantas funcionalidades obtiveram o percentual preestabelecido em cada uma das categorias de funcionalidades. 
Figura 1. Fluxograma do Processo de Proposição de Funcionalidades.

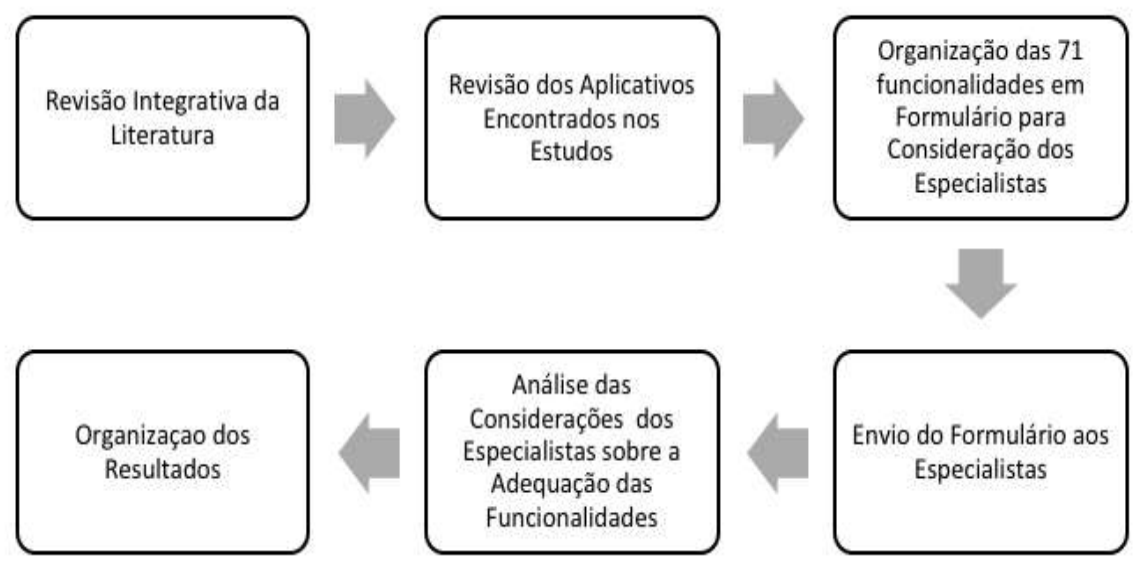

Fonte: Autores da pesquisa (2018).

Como pode ser observado na Figura 1 acima, a partir da Revisão Integrativa da Literatura foi realizado o protocolo de revisão e organização de todos os estudos coletados, resultando na organização dos resultados finais. Antes do início do aplicativo foram criados wireframes de algumas possíveis telas para auxiliar no desenvolvimento, essas telas foram pensadas de maneira em que um usuário pudesse utilizar todas as funcionalidades do aplicativo a partir da tela inicial.

Para o desenvolvimento utilizando as funcionalidades selecionadas pelos especialistas, foi utilizada a biblioteca React em conjunto com o framework React Native, esse framework possibilita o uso de uma única linguagem de programação (JavaScript) em conjunto com linguagens de codificação (HTML e CSS) gerar um aplicativo para os Sistemas Operacionais Android® e iOS®.

\section{Resultados}

Os resultados do formulário enviado aos especialistas foram transcritos para melhor compreensão dos mesmos. Sabese que entre os experts, três são médicos psiquiatras e dois são psicólogos; onde desses, um se intitulou pós-doutor, dois intitularam-se doutores, um intitulou-se professor titular e um especialista.

O formulário continha as funcionalidades separadas por categorias que englobavam as funcionalidades semelhantes. As oito categorias organizadas são: informativos sobre sinais e sintomas psiquiátricos; quiz/checklist; contato de serviços de saúde e saúde mental; recursos para ajuda online/outros recursos online; recursos com vídeos/Imagens; plano de segurança/cartão de crise; recursos para relaxamento/atividades físicas/mindfullness; jogos/passatempos/redes sociais.

Desse modo, foram considerados os resultados que continham $80 \%$ ou mais de consideração de adequação entre os especialistas que responderam o formulário. Assim, sabe-se que na primeira categoria, três funcionalidades estavam dentro do percentual de adequação adotado: dicas para lidar com a ansiedade; sinais de alerta para risco de suicídio; sugestões de como agir em caso de preocupação. Na segunda categoria, duas funcionalidades se enquadraram no padrão estabelecido: questionário avaliativo/checklist de sintomas psiquiátricos; questionário sobre autoagressão. A terceira categoria, por sua vez, possui quatro funcionalidades com $80 \%$ ou mais de consideração de adequação entre os especialistas: mapas indicando centros de saúde ou de saúde mental nas proximidades; contato/linha direta de centros de crise e serviços de saúde mental; contatos de emergência geral e contatos próprios do usuário; direcionamento para chat de serviços de prevenção ao suicídio. A quarta categoria de funcionalidades obteve duas funcionalidades com o percentual mínimo de adequação estabelecido: chat para conversa com pessoas treinadas para lidar com sintomas psiquiátricos; link para site de prevenção ao suicídio. A quinta categoria, por sua 
vez, não teve nenhuma funcionalidade com o mínimo de $80 \%$ de adequação entre os experts. Na sexta categoria uma funcionalidade obteve $80 \%$ ou mais de adequação considerada pelos experts: criar plano de segurança para a prevenção do suicídio. A sétima categoria, no entanto, contou com uma funcionalidade que se encaixa no percentual mínimo estabelecido: guia de relaxamento. A oitava e última categoria incluiu uma funcionalidade dentro do mínimo estabelecido: criar e participar de grupos de apoio no aplicativo.

Observa-se que dentre as funcionalidades que obtiveram 100\% de adequação entre os cinco especialistas estão três: contato/linha direta de centros de crise e serviços de saúde mental; direcionamento para chat de serviços de prevenção ao suicídio; criar plano de segurança para a prevenção do suicídio. Para fins de estudo, sabe-se que as três funcionalidades supracitadas estão contempladas na concordância em $80 \%$ ou mais preestabelecida pelos autores. O número de 15 funcionalidades não obteve indicação pelos especialistas, ou seja, $0 \%$ de concordância quanto a sua adequação a um protótipo de aplicativo de prevenção ao suicídio para a realidade brasileira. Foi obtido com a consideração de adequação de 80\% ou mais descritas nas categorias, um total de 14 funcionalidades.

Para fins de melhor organização do estudo, as funcionalidades resultantes da pesquisa foram agrupadas entre 11 "Funcionalidades de Autogestão (AG)", ou seja, que não exigem contato com outros profissionais e serviços, e seis "Funcionalidades de Gestão Compartilhada (GC)", que necessitam que o usuário tenha contato com outros profissionais e/ou serviços, conforme Quadro 1.

Quadro 1. Lista funcionalidades com $80 \%$ ou mais de concordância entre os juízes.

\begin{tabular}{|c|c|c|}
\hline Identificação & Título da Funcionalidade & App \\
\hline $\mathrm{F} 1-\mathrm{AG}$ & Dicas para lidar com a ansiedade, dor e emoções & Stay Alive \\
\hline $\mathrm{F} 2-\mathrm{AG}$ & Sinais de alerta para risco de suicídio & $\begin{array}{l}\text { Step Up Speak Out; U matter U can get help; } \\
\text { ASK \& prevent suicide; Guard your buddy; } \\
\text { RUOKOSU }\end{array}$ \\
\hline F3 - AG & $\begin{array}{l}\text { Sugestões de como agir em caso de preocupação com } \\
\text { a própria segurança ou de alguém próximo }\end{array}$ & $\begin{array}{l}\text { Step Up Speak Out; U matter U can get help; } \\
\text { Guard your buddy; Stay Alive }\end{array}$ \\
\hline $\mathrm{F} 4-\mathrm{AG}$ & $\begin{array}{llll}\begin{array}{l}\text { Questionário } \\
\text { psiquiátricos }\end{array} & \text { avaliativo/checklist de sintomas } \\
\end{array}$ & $\begin{array}{l}\text { A.L.E.R.T.; U matter U can get help; } \\
\text { Previdence; MoodTools - Depression }\end{array}$ \\
\hline F5 - AG & Questionário sobre autoagressão & Skarz Diary \\
\hline F6 - AG & Link para site de prevenção ao suicídio & RUOKOSU; Wingman Toolkit \\
\hline F7 - AG & $\begin{array}{l}\text { Guia de relaxamento (respiração controlada, } \\
\text { relaxamento muscular, meditação) }\end{array}$ & ReliefLink; Wingman Toolkit \\
\hline F8 - AG & Criar plano de segurança para a prevenção do suicídio & $\begin{array}{l}\text { My Game Plan; Suicide? Help; MY3; } \\
\text { ReliefLink; Stay Alive; MoodTools - } \\
\text { Depression; Suicide Safety Plan }\end{array}$ \\
\hline F9 - GC & $\begin{array}{l}\text { Mapas indicando centros de saúde ou de saúde mental } \\
\text { nas proximidades }\end{array}$ & $\begin{array}{l}\text { U matter U can get help; ReliefLink; Suicide } \\
\text { Safety Plan }\end{array}$ \\
\hline F10 - GC & $\begin{array}{l}\text { Contato/linha direta de centros de crise e serviços de } \\
\text { saúde mental }\end{array}$ & $\begin{array}{l}\text { A.L.E.R.T.; Virtual Hope Box; Operation } \\
\text { Reach Out; Step Up Speak Out; Talklife; U } \\
\text { matter U can get help; ASK \& prevent } \\
\text { suicide; Guard your Buddy; My Game Plan; } \\
\text { Suicide? Help!; Teen Hotlines; RUOKOSU; } \\
\text { MY3; Relieflink; Stay alive; Hello Cruel } \\
\text { World; Wingman Toolkit; Maan-sanvad }\end{array}$ \\
\hline F11 - GC & $\begin{array}{l}\text { Contatos de emergência geral e contatos próprios do } \\
\text { usuário }\end{array}$ & $\begin{array}{l}\text { A.L.E.R.T.; Virtual Hope Box; Operation } \\
\text { Reach Out; MY3 }\end{array}$ \\
\hline F12 - GC & $\begin{array}{l}\text { Direcionamento para chat de serviços de prevenção } \\
\text { ao suicídio }\end{array}$ & ReliefLink \\
\hline $\mathrm{F} 13-\mathrm{GC}$ & $\begin{array}{l}\text { Chat para conversa com pessoas treinadas para lidar } \\
\text { com sintomas de depressão }\end{array}$ & The Hope Line \\
\hline $\mathrm{F} 14-\mathrm{GC}$ & Criar e participar de grupos de apoio no aplicativo & Project Toe \\
\hline
\end{tabular}

Fonte: Autores da pesquisa (2018). 
Desse modo, como ilustrado no Quadro 1 acima, foi possível organizar sistematicamente os aplicativos trabalhados em cada um dos estudos utilizados como base para esta pesquisa, permitindo uma visão clara de todas as ferramentas encontradas (vide coluna “App”). Além disso, para fins ilustrativos dos resultados da pesquisa, foi elaborada uma primeira versão de protótipo de aplicativo do celular com algumas das funcionalidades resultantes da pesquisa. Esse protótipo foi criado a partir da biblioteca React e do framework React Native e está disponível tanto em para Android $®$ quanto para iOS® no ambiente local de testes do desenvolvedor, mas ainda não está disponível para download nas lojas de aplicativos. As figuras abaixo (Figura 2, Figura 3, Figura 4, Figura 5, Figura 6 e Figura 7) são imagens das telas que podem compor o app no caso de desenvolvimento do mesmo.

Figura 2. Tela Inicial.

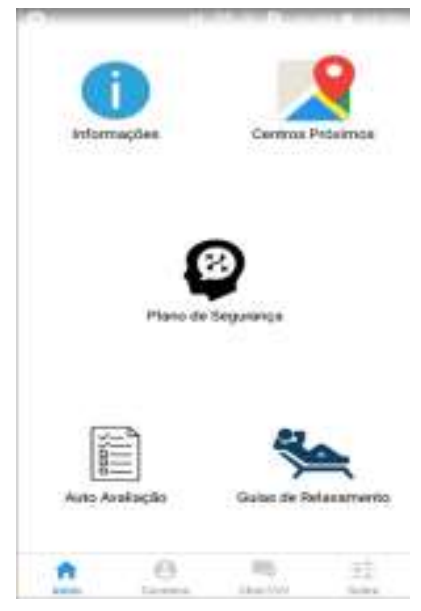

Fonte: Autores da pesquisa (2018).

Figura 4. Plano de Segurança.

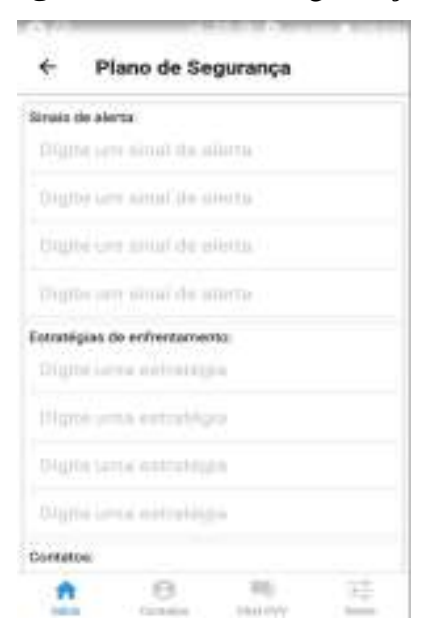

Fonte: Autores da pesquisa (2018).
Figura 3. Contatos de Emergência.

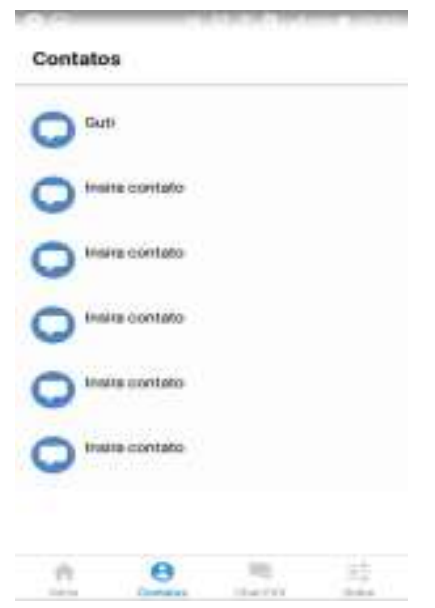

Fonte: Autores da pesquisa (2018).

Figura 5. Auto Avaliação.

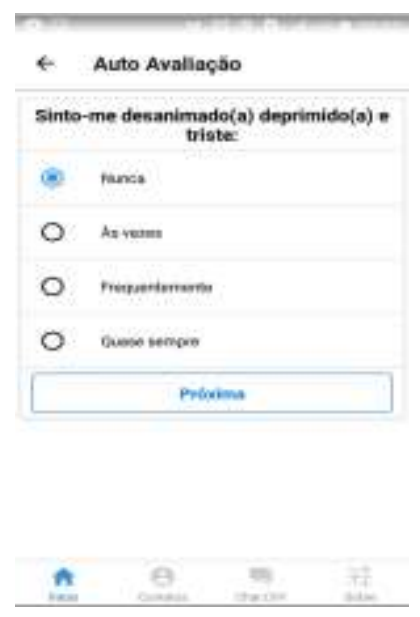

Fonte: Autores da pesquisa (2018). 
Figura 6. Informações.

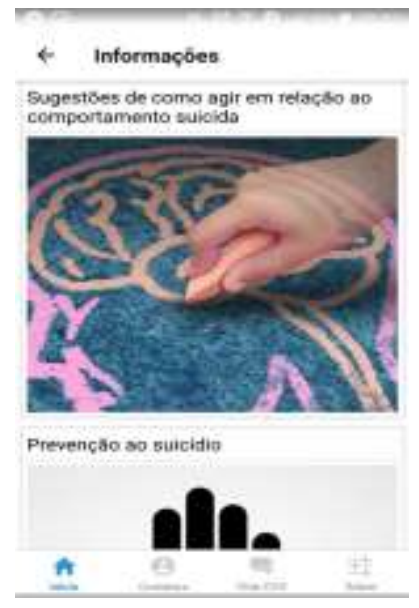

Fonte: Autores da pesquisa (2018).
Figura 7. Guias de relaxamento.

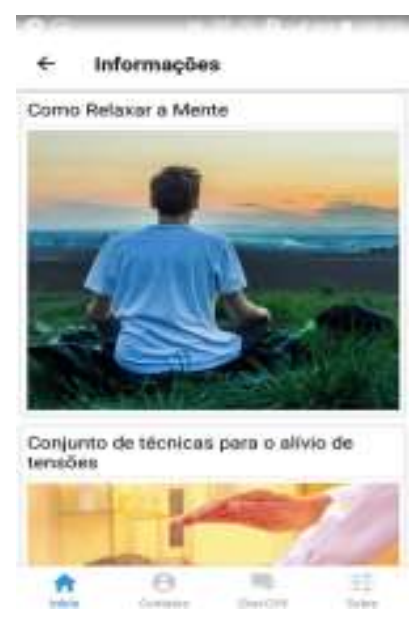

Fonte: Autores da pesquisa (2018).

\section{Discussão}

Para que a prevenção do comportamento suicida seja possível, é preciso situar que existem diversos fatores que influenciam a vida da pessoa que está em sofrimento, como elementos biológicos, psicológicos, genéticos, culturais, sociais e ambientais (Silva, \& Daiuto, 2017). Desse modo, vê-se que é necessário planejamento rigoroso e criação de programas que envolvam diversos profissionais e instituições que trabalhem juntamente com a comunidade na prevenção do comportamento suicida (Silva, \& Daiuto, 2017).

Nesse aspecto, sabe-se que o Ministério da Saúde do Brasil possui e instituiu em 2006 as Diretrizes Nacionais para Prevenção do Suicídio, onde propõe, entre outras ações, que se desenvolvam estratégias de promoção da qualidade de vida, educação e proteção e recuperação da saúde e prevenção de danos; que se organizem linhas de cuidados integrais em todos os níveis de atenção, garantindo o acesso às diferentes modalidades terapêuticas e que se fomente e executem projetos estratégicos fundamentados em estudos de custo-efetividade, eficácia e qualidade, bem como em processos de organização da rede de atenção e intervenções no caso de tentativas de suicídio (Brasil, 2006).

Com isso, pode-se considerar o desenvolvimento de um aplicativo de celular para a prevenção do comportamento suicida como uma estratégia complementar às demais políticas instituídas e serviços oferecidos. Então, vê-se que num modelobase de aplicativo de celular para esse fim, podem estar presentes funcionalidades que envolvam ou não instituições e serviços de saúde ou profissionais da área, bem como funcionalidades que sejam de uso individual da pessoa que busca recursos em momento de crise e/ou sofrimento. É importante que se diferenciem, nesse aspecto, as funcionalidades de autogestão do cuidado e as funcionalidades de gestão compartilhada ou cogestão do cuidado a pessoa em relação à prevenção do comportamento suicida.

Nesse sentido, sabe-se que a autonomia é uma das bases do processo de desinstitucionalização e da reforma psiquiátrica e, sua conquista, pode ser contemplada com o processo do cuidado clínico dos sujeitos (Alves, et al., 2018). Esse processo de possibilitar e fortalecer a autonomia pode se dar a partir da construção de espaços e dispositivos de cuidado horizontalizados, bem como através do acolhimento, da co-responsabilização e da promoção da autonomia da pessoa (Gonçalves, \& Campos, 2017; Jorge et al., 2011).

Com isso, vê-se que algumas das funcionalidades consideradas adequadas pelos experts dependem unicamente do usuário para serem utilizadas, como, por exemplo, o acesso às dicas para lidar com a ansiedade, as sugestões sobre como agir em caso de preocupação, a criação do plano de segurança. Quanto às funcionalidades que possuem a proposta de serem utilizadas apenas pelo usuário, reforça-se a necessidade de as mesmas buscarem embasamento científico, ou seja, que sejam 
fundamentadas em literatura e/ou experiências científicas que comprovem a pertinência de seu conteúdo. No caso da funcionalidade "plano de segurança", por exemplo, sabe-se que a mesma, como foi encontrada nos apps estudados, aparece como uma função de autogestão. Entretanto, no Brasil, recomenda-se que os planos de segurança ou de crise sejam elaborados juntamente com o profissional de referência/responsável pelo usuário, colaborando no aprimoramento do mesmo para que seja utilizado como recurso em momentos de crise (Botega, 2015).

Por outro lado, as funcionalidades F9 a F14 caracterizam-se como gestão compartilhada, ou cogestão, ou seja, envolvem serviços e/ou profissionais de saúde para serem utilizadas pelo usuário. Desse modo, é importante que se saiba que na atenção psicossocial, a troca de experiências e a corresponsabilização entre os atores envolvidos no cuidado são de fundamental importância para promover a cidadania, o protagonismo e a autonomia do usuário em saúde mental. Nesse aspecto são envolvidos o usuário, a família e a equipe multiprofissional que tem participação no cuidado do mesmo, por vezes, no Projeto Terapêutico Singular (Lima, Aguiar, \& Souza, 2015).

Além disso, a cogestão do cuidado engloba ainda o autocuidado apoiado, que trata de trabalhar de modo com que as habilidades e confiança das pessoas em gerenciar sua saúde sejam ampliadas. Desse modo é estabelecida uma relação entre equipe de saúde e usuário onde com o apoio dos profissionais, o usuário é quem decide quais comportamentos quer adotar no momento, pactuando e elaborando um plano para isso com a equipe de saúde, que acompanhará e monitorará o processo, auxiliando na adequação do plano quando necessário (Curitiba, 2018).

Como exemplos dessa gestão compartilhada do cuidado está, no Brasil, o Centro de Valorização da Vida (CVV), que oferece contato/linha direta aos usuários, bem como disponibiliza chat para conversas com voluntários treinados para realizar escuta, apoio emocional e prevenção do suicídio às pessoas em momentos de sofrimento (CVV, 2017).

Nesse aspecto, é importante que se reflita acerca da realidade do local a ser desenvolvido o app para a prevenção do comportamento suicida, uma vez que nas funcionalidades de gestão compartilhada do cuidado sejam oferecidos serviços disponibilizados pelo sistema de saúde local, como no caso da funcionalidade "mapas indicando centros de saúde ou saúde mental nas proximidades" e em "contatos de emergência geral e contatos próprios do usuário". A adequação do aplicativo de celular à realidade sanitária dos municípios no Brasil é fundamental também para que se ofereçam recursos adequados ao perfil dos usuários locais.

Sabe-se, ainda, a partir da revisão dos aplicativos realizada, que a média de funcionalidades por app é de cinco, ou seja, as 14 funcionalidades resultantes da consideração dos especialistas são uma base para que se possam escolher quais as funcionalidades mais adequadas à cada realidade sanitária. O desenvolvedor pode avaliar o contexto do local onde lançará o aplicativo e pode adequá-lo conforme a necessidade, se não for possível oferecer recursos extra-app, existem as funcionalidades de autogestão do cuidado, porém se o mesmo possuir como recurso profissionais e serviços de saúde, pode oferecer também as seis funcionalidades de gestão compartilhada do cuidado.

\section{Conclusão}

O estudo identificou 14 funcionalidades resultado de uma pesquisa qualitativa que envolveu revisão integrativa de literatura e consulta à especialistas na área de prevenção ao suicídio. Foi possível indicar um modelo de aplicativo base/de referência a partir de funcionalidades estudadas para possibilitar o desenvolvimento de aplicativos de celular para a prevenção do comportamento suicida no Brasil.

Estas funcionalidades foram identificadas como Autogestão do usuário e Gestão Compartilhada entre usuário e profissionais de serviços de saúde mental. Isto significa que estas funcionalidades podem ser adequadas conforme a realidade subjetiva da pessoa e da realidade sanitária local do sistema de saúde. Com este recurso tem-se uma possibilidade a mais para o campo da prevenção do comportamento suicida. 
Reconhece-se que este app poderá fazer parte de pesquisas sobre a prevenção do comportamento suicida e, ao ser incluído, possa ser avaliado se contribui, ou não, como uma variável na prevenção do comportamento suicida, que é multifatorial. Sugere-se, portanto, novos estudos que abordem o uso de aplicativos para esse fim, buscando corroborar o presente estudo e, principalmente ampliar o acesso aos cuidados em saúde mental pelos usuários em situação de sofrimento, validando a sua utilidade e importância nos momentos de crise.

\section{Referências}

Alves, P. F., Kantorski, L. P., Andrade, A. P. M., Coimbra, V. C. C., Oliveira, M. M., \& Silveira, K. L. (2018). Ser autônomo: o que os serviços de saúde mental indicam? Rev. Gaúcha Enferm. 1(39), 1-17. https://www.scielo.br/scielo.php?script=sci_arttext\&pid=S1983-14472018000100420. doi. https://doi.org/10.1590/1983-1447.2018.63993.

Band, R. J. (2013). Significant others, patient outcomes and maintenance of symptoms in chronic fatigue syndrome. (Tese de Doutorado). Curso de School Of Psychological Sciences, Faculty of Medical and Human Sciences, University Of Manchester, Manchester.

Botega, N. J., Cais, C. F. S., Correa, H., Segal, J., Stefanello, J. A. C. J. M. B. S. Comportamento Suicida: Conhecer para prevenir. São Paulo: Associação Brasileira de Psiquiatria, 2009. Recuperado de http://www.sauesp.org.br/cse003/ComportamentoSuicidaConhecerParaPrevenir.pdf.

Botega, N. J. (2015). Crise Suicida: Avaliação e Manejo. Artmed, 302 p.

Boulos, M. N. K., Wheeler, S., Tavares, C., \& Jones, R. (2011). How smartphones are changing the face of mobile and participatory healthcare: an overview, with example from eCAALYX. Biomedical Engineering Online, 10(1), 1-14. https://biomedical-engineering-online.biomedcentral.com/articles/10.1186/1475925X-10-24. doi.10.1186/1475-925x-10-24.

Brasil. Portaria $n^{o}$ 1.876, de 14 de agosto de 2006. Diretrizes Nacionais Para Prevenção do Suicídio. http://bvsms.saude.gov.br/bvs/saudelegis/gm/2006/prt1876_14_08_2006.html.

CVV (2017). Centro de Valorização da Vida. https://www.cvv.org.br.

Curitiba, Secretaria Municipal de Saúde. Autocuidado apoiado. (2018). http://www.saude.curitiba.pr.gov.br/programas/saude-adulto/autocuidado.html.

Durkheim, E. (2008). El suicídio. (6a ed). Akal.

Fjeldsoe, B. S., Marshall, A. L., \& Miller, Y. D. (2009). Behavior change interventions delivered by mobile telephone short-message service. american Journal Of Preventive Medicine, 36(2), 165-173. https://www.ncbi.nlm.nih.gov/pubmed/19135907. 10.1016/j.amepre.2008.09.040.

Gonçalves, L. L. M., \& Campos, R. T. O. (2017). Narrativas de usuários de saúde mental em uma experiência de gestão autônoma de medicação. Cadernos de Saúde Pública, 33(11), 1-11. https://www.scielosp.org/article/csp/2017.v33n11/e00166216/pt/. 10.1590/0102-311x00166216.

Jorge, M. S. B., Pinto, D. M., Quinderé, P. H. D., Pinto, A. G. A., Sousa, F. S. P., \& Cavalcante, C. M. (2011). Promoção da Saúde Mental - Tecnologias do Cuidado: vínculo, acolhimento, co-responsabilização e autonomia. Ciência \& Saúde Coletiva, 7(17), 3051-3060. https://www.scielosp.org/pdf/csc/201 1.v16n7/305 1-3060/pt. https://doi.org/10.1590/S1413-81232011000800005.

Lima, M. S., Aguiar, A. C. L., \& Sousa, M. M. (2015). O cuidado compartilhado em saúde mental como potencial de autonomia do usuário. Psicologia em Estudo, 20(4), 675-686. http://www.periodicos.uem.br/ojs/index.php/PsicolEstud/article/view/28309/pdf. 10.4025/psicolestud.v20i4.28309.

Padurariu, M., Prepelita, R., Ciobica, A., Dobrin, R., Timofte, D., Stefanescu, C., \& Chirita R. (2016). Concept of Suicide: Neurophysiological/Genetic Theories and Possible Oxytocin Relevance. Neurophysiology, 48(4), 312-321. https://link.springer.com/article/10.1007/s11062-016-9603-9\#citeas. 10.1007/s11062-016-9603-9.

Pompeo, D. A., Rossi, L. A., \& Galvão, C. Revisão integrativa: etapa inicial do processo de validação de diagnóstico de enfermagem. Acta Paulista de Enfermagem, 22(4), 434-438. https://www.scielo.br/scielo.php?pid=s0103-21002009000400014\&script=sci_abstract\&tlng=pt 10.1590/s010321002009000400014

Pop-Eleches, C., Thirumurthy, H., Habyarimana, J. P., Zivin, J. G., Goldstein, M. P., Walque, D., MacKeen, L., et al., Bangsberg, D. R.. Mobile phone technologies improve adherence to antiretroviral treatment in a resource-limited setting: a randomized controlled trial of text message reminders. (2011). Aids, 25(6), 825-834. https://www.ncbi.nlm.nih.gov/pmc/articles/PMC3718389/. 10.1097/qad.0b013e32834380c1.

Silva, E. H. M. S., \& Daiuto, P. R. (2017). O atendimento clínico ao cliente com comportamento suicida: desafios e possibilidades. Revista Uningá, 52(1), 157-161. http://revista.uninga.br/index.php/uninga/article/view/1373.

Werlang, B. S. G., Borges, V. R., \& Fensterseifer, L. (2005). Fatores de risco ou proteção para a presença de ideação suicida na adolescência. Revista Interamericana de Psicologia, 2(39), 259-266. https://www.redalyc.org/articulo.oa?id=28439210.

WHO, World Health Organization. (2014). Preventing Suicide: A global Imperative. http://apps.who.int/iris/bitstream/handle/10665/131056/97892415647 79_eng.pdf;jsessionid=8279081F5F543FD90523899CB416B615? sequence=1\%3E.

WHO, World Health Organization. (2006). Prevenção do Suicídio: Um recurso para conselheiros. Genebra: OMS, 28p. http://apps.who.int/iris/bitstream/handle/10665/43487/9241594314_eng.pdf;jsessionid=D079DDB629F5D0C753AD8E0C8F5A8978?sequence=1. 